\title{
Emergency attendances and hospitalisations for complications after transrectal ultrasound- guided prostate biopsies: a five-year retrospective multicentre study
}

\author{
KC Cheng *, WC Lam, HC Chan, CC Ngo, MH Cheung, HS So, KM Lam
}

\section{A B S T R A C T}

Introduction: Transrectal ultrasound-guided (TRUS) prostate biopsy is an established procedure for diagnosis of prostate cancer. Complications after TRUS biopsy are not well reported in Hong Kong. This study evaluated the 5-year incidences of TRUS biopsy complications and potential risk factors for those complications.

Methods: This was a retrospective review of biopsies performed from 2013 to 2017 in two local hospitals using data retrieved from electronic medical records. The primary outcome was the occurrence of complications requiring either emergency attendances or hospitalisations within 30 days after biopsy. Potential risk factors were examined using multiple logistic regression analysis.

Results: In total, 1699 men were included (mean age \pm standard deviation: $67 \pm 7$ years; median prostatespecific antigen level: $7.9 \mu \mathrm{g} / \mathrm{L}$ [interquartile range, $5.5-12.6 \mu \mathrm{g} / \mathrm{L}]) ; 4.3 \%$ had pre-biopsy bacteriuria. Overall, $5.7 \%$ and $3.8 \%$ of post-biopsy complications required emergency attendances and hospitalisations, respectively. Gross haematuria and rectal bleeding requiring emergency attendances developed in $2.1 \%$ and $0.4 \%$ of men; $0.8 \%$ and $0.4 \%$ required hospitalisations. Furthermore, $1.5 \%$ of men developed acute urinary retention requiring

This article was published on 11 Oct 2019 at www.hkmj.org. hospitalisations; $1.9 \%$ and $1.2 \%$ had post-biopsy infections requiring emergency attendances and

hospitalisations, respectively, and $0.9 \%$ had urosepsis requiring hospitalisations. Prostate volume $>48 \mathrm{cc}$ was associated with an increased risk of post-biopsy retention (odds ratio 2.75, 95\% confidence interval: 1.23-4.17)

Conclusions: The rate of overall complications after TRUS biopsy was low. The most common complications requiring emergency attendances and hospitalisations were gross haematuria and acute urinary retention, respectively. Prostate volume $>48 \mathrm{cc}$ increased the risk of post-biopsy urinary retention.

New knowledge added by this study

- Complications requiring emergency attendances or hospitalisations after transrectal ultrasound-guided (TRUS) prostate biopsies are uncommon.

- The most common complications requiring emergency attendances and hospitalisations are gross haematuria and acute urinary retention, respectively.

- The presence of a large prostate (volume $>48 \mathrm{cc}$ ) increases the risk of acute urinary retention after TRUS biopsy. However, no specific factors are associated with increased risk of post-biopsy infections.

Implications for clinical practice or policy

- Patients with large prostate should be counselled for the increased risk of urinary retention after TRUS biopsy.

- Despite the presence of antibiotic-resistant bacteria in urine and blood cultures, patients who develop sepsis after TRUS biopsy are likely to recover after a brief period of hospitalisation.

\section{Introduction}

Transrectal ultrasound-guided (TRUS) prostate biopsy, introduced in 1989, ${ }^{1}$ is an established and longstanding procedure for detection of prostate cancer. Because it can be learned rapidly and comprises a simple, office-based procedure, TRUS biopsy remains the most commonly performed procedure for diagnosis of prostate cancer., ${ }^{2,3}$ 


\section{經直腸前列腺穿刺活組織檢查併發症：五年期多 中心回顧性調查}

鄭冠中、林穎聰、陳開澤、敖章鐘、張文虹、蘇慶成、林建文

引言：經直腸前列腺穿刺活組織檢查是診斷前列腺腫瘤的有效方法。 不過, 在香港有關檢查引起併發症的具體資料並不多。本研究審視香 港兩間醫院五年內發生的活檢後併發症, 並嘗試找出引起併發症的風 險因素。

方法：這項回顧性研究審視由2013年至2017年在兩間公立醫院所做的 活檢。主要研究結果包括活檢後 30 天內的急症求診率及住院率。研究 利用多元邏輯斯迴歸分析檢視潛在的併發症風險因素。

結果：1699名男性被納入研究, 平均年齡67歲（標準差：7歲）, 前 列腺特異抗原指數的中位數為 $7.9 \mu \mathrm{g} / \mathrm{L}$ （四分位距：5.5-12.6 $\mathrm{g} / \mathrm{L}$ ）， 當中 $4.3 \%$ 患者活檢前小便帶菌。30天內急症求診率和住院率分別為 $5.7 \%$ 及 $3.8 \%$ 。因血尿和便血到急症室求診的佔 $2.1 \%$ 和 $0.4 \%$, 其中分 別有 $0.8 \%$ 及 $0.4 \%$ 患者須住院。急性尿潴留患者佔 $1.5 \%$, 全部須住院。 因活檢後感染到急症室求診的患者佔 $1.9 \%$, 須住院的佔 $1.2 \%$ 。因感染 引致敗血症的住院率為 $0.9 \%$ 。前列腺體積超過 $48 \mathrm{cc}$ 的患者在活檢後引 發尿噭留的機會較高（比值比：2.75，95\%置信區間：1.23-4.17）。

結論：經直腸前列腺穿刺組織檢查的併發症低。最常見因併發症往急 症室求診及住院的分別是血尿及尿潴留。前列腺體積超過 $48 \mathrm{cc}$ 的患 者, 活檢後引發尿濐留的機會較高。

However, TRUS biopsy is associated with significant risks. Instances of bleeding are common, including haematuria, rectal bleeding, and haemospermia; however, these are generally mild and self-limiting. ${ }^{4}$ The most worrisome complication is post-biopsy infection, which occurs in $0 \%$ to $6.3 \%$ of men after TRUS biopsy. ${ }^{4}$ The risk is low, but the consequences are serious in affected patients. There is recent evidence to suggest that increasing numbers of quinolone-resistant organisms are contributing to the development of post-biopsy sepsis. ${ }^{4}$

In Hong Kong, there have been few reports of TRUS biopsy complications. Some studies have focused on infective complications in relatively small numbers of patients. ${ }^{5,6}$ Therefore, we reviewed TRUS biopsies performed over a 5-year period in two local hospitals to evaluate the incidences and types of complications, as well as their associated risk factors. This could provide an important insight into the overall TRUS biopsy complications, including infective and non-infective complications in the local population.

\section{Methods}

\section{Patients and study design}

This retrospective cohort analysis included men who underwent TRUS biopsy procedures during the period from 2013 to 2017 in United Christian Hospital, Hong Kong and Tseung Kwan O Hospital, Hong Kong. All patients who underwent TRUS biopsy procedures were included in the analysis.
Indications for biopsy included elevated prostatespecific antigen (PSA) level, suspicious digital rectal examination of the prostate, restaging biopsies in incidental prostate cancer detected in transurethral prostatectomy or in patients under active surveillance of prostate cancer, and previous suspicion of prostate cancer (eg, high-grade prostate intraepithelial neoplasia or atypical small acinar proliferation). Pre-biopsy blood tests were performed to determine complete blood count, clotting profile, and PSA level. Mid-stream urine was collected 3 to 4 weeks prior to biopsy for bacterial culture analysis. A course of antibiotic treatment was administered if pre-biopsy bacteriuria was detected, based on the sensitivity profile of the involved bacteria. Anticoagulant medications and clopidogrel were discontinued prior to biopsy; the duration of cessation and any requirement for heparin coverage were determined by physicians. The use of low-dose aspirin was continued during biopsy. Oral bisacodyl tablets were used for rectal preparation on the morning of the biopsy procedure. Quinolone antibiotic prophylaxis with oral levofloxacin $500 \mathrm{mg}$ was prescribed 1 hour prior to biopsy, then continued for 2 days after biopsy. This report was compiled in accordance with the STROBE guidelines. ${ }^{7}$ The principles outlined in the Declaration of Helsinki were followed.

\section{Biopsy procedure}

All biopsies were performed as day procedures. A 7.5-MHz biplanar transrectal ultrasound probe and 18-gauge needles with side-firing needle-guides were used for biopsy. Each patient was positioned in the left lateral posture with both hips and knees flexed. Prostate size measurement was calculated using the ellipsoidal formula. Topical lidocaine jelly and local anaesthetic injection with $10 \mathrm{~mL}$ of $1 \%$ plain lidocaine were used routinely in one hospital; these were injected into the area between the prostatic base and seminal vesicles. The other hospital used topical lidocaine alone. Six-core to 12-core systemic biopsies were performed depending on the hospital involved and the time frame of the biopsy procedure, as the two centres have changed the practice in performing more number of cores with time. Each patient was discharged on the same day after completion of the procedure. Clinical follow-up was performed at 4 weeks post-biopsy in an out-patient clinic to review the pathology findings.

\section{Follow-up assessment}

Patients who were admitted for biopsies were identified using the Clinical Data Analysis and Reporting System. Clinical records (ie, discharge summary, emergency case notes, clinic consultation notes, laboratory results, and ultrasound findings) were retrieved using the hospital-based Clinical Management System and the territory-wide 
Electronic Patient Record, which comprises a centralised medical records system shared by all public hospitals. Thus, men who had been admitted to another public hospital for complications could be identified. The patients' records were examined and the occurrence of complications was determined using a standardised form. During post-biopsy follow-up examinations, clinical records from the Clinical Management System were examined to identify any potential attendances or admissions to private sector hospitals owing to complications. The primary outcome in this study was the occurrence of complications within 30 days after biopsy. Complications were defined as events requiring either emergency attendances or hospitalisations; these events were analysed separately. Post-biopsy urinary tract infections (PBI) were defined as the presence of urinary tract infection symptoms (dysuria, with or without frequency, urgency, or suprapubic pain) after biopsy, with or without sepsis. Based on the Sepsis-3 criteria, sepsis was defined as an acute increase in the Sequential Organ Failure Assessment score of $\geq 2 .{ }^{8}$ Acute urinary retention (AUR) was defined as acute painful retention of urine requiring catheterisation. Any lower urinary tract symptoms (LUTS) that occurred or worsened after biopsy, which required emergency attendances, were also recorded.

\section{Statistical analysis}

Statistical calculations were computed with the SPSS (Windows version 22.0; IBM Corp, Armonk [NY], United States). For examination of potential risk factors, continuous variables, such as PSA level and prostate size, were categorised based on the median values. The Chi squared test was used to compare complications between the two hospitals. Multiple logistic regression models were used to investigate potential risk factors for complications.

\section{Results}

In total, 1710 men were admitted to either of the two hospitals for TRUS biopsy procedures during the study period. Eleven men were excluded because they refused to undergo TRUS biopsy after admission; therefore, 1699 men were included in the study. The mean age ( \pm standard deviation) of the men was $67 \pm 7$ years and median PSA level was $7.9 \mu \mathrm{g} / \mathrm{L}$ (interquartile range, 5.5-12.6 $\mu \mathrm{g} / \mathrm{L}$ ). Of the 1699 men in the study, 310 (18.2\%) had a suspicious digital rectal examination of the prostate; the overall cancer detection rate was $19.8 \%$. Characteristics and results of the biopsies are shown in Table 1. Overall, $5.7 \%$ and $3.8 \%$ of post-biopsy complications required emergency attendances and hospitalisations, respectively (Table 2 ). There were no occurrences of mortality in the entire cohort.
TABLE I. Characteristics and results of prostate biopsies $(n=1699)^{*}$

\begin{tabular}{lr}
\hline & \multicolumn{1}{c}{ Value } \\
\hline Hospital & \\
\hline Centre 1 & $960(56.5 \%)$ \\
\hline Centre 2 & $739(43.5 \%)$ \\
\hline Indications & \\
\hline Elevated PSA & 1334 (78.5\%) \\
\hline Abnormal DRE & $98(5.8 \%)$ \\
\hline Elevated PSA and abnormal DRE & $159(9.4 \%)$ \\
\hline Restaging biopsies & $44(2.6 \%)$ \\
\hline $\begin{array}{l}\text { History of suspicious biopsies/HGPIN/ } \\
\text { ASAP }\end{array}$ & $59(3.5 \%)$ \\
\hline Incidental prostate lesions in imaging & $5(0.3 \%)$ \\
\hline Operators & \\
\hline
\end{tabular}

$\begin{array}{lr}\text { Radiologists or trainees } & 372(21.9 \%) \\ \text { Urologists or trainees } & 1327(78.1 \%)\end{array}$

Antiplatelet/anticoagulation

Low-dose aspirin $\quad 305$ (18.0\%)

Heparin/low-molecular-weight heparin $8(0.5 \%)$

Periprostatic nerve block

Yes

793 (46.7\%)

No

906 (53.3\%)

Episodes of biopsies

First

1149 (67.6\%)

Second

$440(25.9 \%)$

Third

$91(5.4 \%)$

Fourth or more

$19(1.1 \%)$

No. of cores

$<6$

$10(0.6 \%)$

6

329 (19.4\%)

8

493 (29.0\%)

10

$530(31.2 \%)$

12

323 (19.0\%)

$>12$

$3(0.2 \%)$

Not documented

$11(0.6 \%)$

Prostate size, cc, median (interquartile range)

48 (34-66)

Pathology

Benign

$1263(74.3 \%)$

Acinar adenocarcinoma

$335(19.7 \%)$

Ductal adenocarcinoma

$1(0.1 \%)$

HGPIN

$29(1.7 \%)$

ASAP

$38(2.2 \%)$

Prostatitis

$33(1.9 \%)$

Abbreviations: ASAP = atypical small acinar proliferation; DRE = digital rectal examination; HGPIN = high-grade prostate intraepithelial neoplasia; PSA = prostate-specific antigen * Data are shown as No. (\%), unless otherwise specified 


\section{Bleeding complications}

Overall, $2.1 \%$ of men in the study developed gross haematuria requiring emergency attendances, and $0.8 \%$ were hospitalised for further management. Haematuria subsided with conservative treatment in all affected men; no transfusions or emergency surgical interventions were needed. Rectal bleeding occurred in $0.4 \%$ of men; all required hospitalisations. Rectal bleeding resolved spontaneously in all affected men, except two who required rectal packing with adrenaline gauze for haemostasis. There were no cases of haemospermia requiring emergency attendances. No risk factors could be identified

TABLE 2. Complications requiring emergency attendances or hospitalisations after prostate biopsies $(\mathrm{n}=1699)^{*}$

\begin{tabular}{lcc}
\hline & Emergency attendances & Hospitalisations \\
\hline Overall & $96(5.7 \%)$ & $65(3.8 \%)$ \\
Infective complications & & \\
$\quad$ Overall PBI & $32(1.9 \%)$ & $20(1.2 \%)$ \\
Sepsis & $15(0.9 \%)$ & $15(0.9 \%)$ \\
Non-infective complications & & \\
$\quad$ Haematuria & $35(2.1 \%)$ & $14(0.8 \%)$ \\
Rectal bleeding & $6(0.4 \%)$ & $6(0.4 \%)$ \\
Haemospermia & 0 & 0 \\
\hline AUR & $25(1.5 \%)$ & $25(1.5 \%)$ \\
LUTS & $7(0.4 \%)$ & $2(0.1 \%)$ \\
\hline
\end{tabular}

Abbreviations: AUR = acute urinary retention; LUTS = lower urinary tract symptoms; $\mathrm{PBI}=$ post-biopsy infection

* Data are shown as No. (\%) for emergency attendances or hospitalisations related to any bleeding complications (Table 3). Importantly, the continuation of low-dose aspirin was not associated with an increased rate of bleeding complications.

\section{Retention of urine and lower urinary tract symptoms}

In all, $1.5 \%$ of men in the study developed AUR; all required hospitalisations. During these hospitalisations, the men were assessed by voiding trials; all were able to void spontaneously within 2 to 3 days. Acute-onset LUTS was present in $0.4 \%$ of men who had emergency attendances, and $0.1 \%$ of the men required hospitalisation. Prostate size $>48$ cc was associated with a nearly 3 -fold increase in the risk of post-biopsy retention (odds ratio $=2.75$, 95\% confidence interval: 1.23-4.17; Table 3). No risk factors were identified with respect to the occurrence of LUTS.

\section{Post-biopsy infection}

Pre-biopsy bacteriuria was present in $4.3 \%$ of men in this study. The most common causative bacterial species was Escherichia coli (1.8\%) [Table 4]. Emergency attendances and hospitalisation rates for PBI were $1.9 \%$ and $1.2 \%$, respectively. Sepsis occurred in $0.9 \%$ of men in this study, all of whom required hospitalisations (Table 2). Among patients who developed sepsis, none had a positive prebiopsy urine culture. Post-sepsis urine cultures were positive in $46.7 \%(7 / 15)$ of the men who developed sepsis; all of these positive cultures showed growth of $E$ coli, and 57\% (4/7) of the cultures demonstrated quinolone resistance. Blood cultures were positive in

TABLE 3. Multiple logistic regression model examining risk factors for non-infective complications

\begin{tabular}{|c|c|c|c|c|c|c|}
\hline & \multicolumn{4}{|c|}{ Haematuria } & \multirow{2}{*}{\multicolumn{2}{|c|}{$\begin{array}{c}\text { AUR } \\
\begin{array}{c}\text { Emergency attendance/ } \\
\text { hospitalisation }\end{array}\end{array}$}} \\
\hline & \multicolumn{2}{|c|}{ Emergency attendance } & \multicolumn{2}{|c|}{ Hospitalisation } & & \\
\hline & $P$ value & OR $(95 \% \mathrm{Cl})$ & $P$ value & OR $(95 \% \mathrm{Cl})$ & $P$ value & OR $(95 \% \mathrm{Cl})$ \\
\hline Age & 0.17 & $0.96(0.91-1.01)$ & 0.68 & $1.02(0.94-1.11)$ & 0.84 & $1.01(0.94-1.09)$ \\
\hline Diabetes mellitus & 0.24 & $1.22(0.93-1.45)$ & 0.46 & $0.96(0.72-1.23)$ & 0.32 & $1.56(0.90-2.1)$ \\
\hline$P S A \geq 8$ & 0.87 & $0.94(0.43-2.03)$ & 0.62 & $0.73(0.21-2.52)$ & 0.39 & $0.61(0.20-1.89)$ \\
\hline Prostate size $>48 \mathrm{cc}$ & 0.68 & $1.18(0.54-2.57)$ & 0.84 & $1.23(0.35-3.59)$ & 0.04 & $2.75(1.23-4.17)$ \\
\hline Pre-biopsy bacteriuria (treated) & 0.78 & $1.23(0.30-5.07)$ & 0.16 & $3.71(0.61-22.80)$ & 0.26 & $0.30(0.04-2.43)$ \\
\hline Low-dose aspirin & 0.54 & $1.24(0.78-1.45)$ & 0.34 & $1.45(0.82-1.65)$ & 0.32 & $2.12(0.54-4.21)$ \\
\hline Periprostatic nerve block & 0.16 & $0.34(0.08-1.53)$ & 0.12 & $0.19(0.02-1.56)$ & 0.53 & $0.62(0.14-2.73)$ \\
\hline No. of cores $\geq 10$ & 0.56 & $1.57(0.35-7.01)$ & 0.31 & $2.64(0.40-17.45)$ & 0.12 & $3.24(0.72-14.47)$ \\
\hline Repeated biopsies & 0.62 & $0.82(0.36-1.84)$ & 0.46 & $0.60(0.16-2.30)$ & 0.61 & $0.75(0.25-2.26)$ \\
\hline Prostate cancer & 0.25 & $2.09(0.59-7.38)$ & 0.66 & $0.73(0.19-2.84)$ & 0.17 & $0.46(0.16-1.38)$ \\
\hline
\end{tabular}

Abbreviations: $A \cup R=$ acute urinary retention; $\mathrm{Cl}=$ confidence interval; $\mathrm{OR}=$ odds ratio; $\mathrm{PSA}=$ prostate-specific antigen 
TABLE 4. Types of pre-biopsy bacteriuria*

\begin{tabular}{lr}
\hline Bacteria & No. (\%) \\
\hline Citrobacter species & $5(0.3 \%)$ \\
Escherichia coli & $30(1.8 \%)$ \\
Enterococcus species & $8(0.5 \%)$ \\
Klebsiella pneumoniae & $9(0.5 \%)$ \\
Proteus mirabilis & $15(0.9 \%)$ \\
Pseudomonas aeruginosa & $5(0.3 \%)$ \\
\hline
\end{tabular}

* Data are shown as No. (\%)

$40 \%(6 / 15)$ of the men who developed sepsis; all of these positive cultures showed growth of $E$ coli, and $83 \%(5 / 6)$ of the cultures demonstrated quinolone resistance. None of the men required intensive care and none developed prostate abscesses. The median hospital stay for men with sepsis was 6 days (interquartile range, 4-10 days).

Treatment for bacteriuria and the presence of diabetes mellitus both showed no associations with overall infection or urosepsis. No other factors tested including age and prostate size were associated with infective complications. There were no differences in the rates of overall complications requiring either emergency attendances $(6.5 \%$ vs $4.6 \%, \mathrm{P}=0.10)$ or hospitalisations $(3.9 \%$ vs $3.8 \%, \mathrm{P}=0.95)$ between the two hospitals. Moreover, there were no differences in the rates of overall post-biopsy infection or sepsis ( $0.8 \%$ vs $1.6 \%, \mathrm{P}=0.13$ and $0.5 \%$ vs $1.4 \%, \mathrm{P}=0.19$ ).

\section{Discussion}

\section{Non-infective complications}

Non-infective complications after TRUS biopsy were common in this study; fortunately, most comprised minor complications that did not require additional treatment. Using questionnaires and telephone for follow-up of patients who underwent TRUS biopsy, the ProtecT Study group found that haematuria occurred in $65.8 \%$, rectal bleeding occurred in $36.8 \%$, and haemospermia occurred in $92.6 \%$, within 35 days after biopsy. ${ }^{9}$ A recent systematic review of TRUS biopsy complications reported wider ranges of complication rates: haematuria in $27.9 \%$ to $64.5 \%$ of patients, haemospermia in $6 \%$ to $90.1 \%$ of patients, and rectal bleeding in $11.5 \%$ to $40 \%$ of patients. ${ }^{4}$ These wide ranges of complication rates were largely dependent on the methods by which the complications were registered. In our study, the reported bleeding rate was lower, as we only included patients with complications requiring emergency attendances. The differences in our findings suggest that post-biopsy bleeding might generally be mild; thus, it does not require medical consultation.

Prostate size is reportedly associated with the risk of haematuria after biopsies, as is the number of cores, although this particular point remains controversial. ${ }^{10-12}$ However, our study did not find evidence to support these relationships. The postbiopsy retention rate in our study was comparable with that in the literature $(0.2 \%-1.7 \%) .{ }^{4}$ All men had successful voiding trials in our cohort and did not require surgical intervention. Importantly, we found that prostate size was a risk factor for post-biopsy retention, consistent with the results of two other studies. ${ }^{10,11}$

\section{Infective complications}

Infective complications requiring hospitalisation have been reported in $0 \%$ to $6.3 \%$ of patients after TRUS biopsy. 4 The Global Prevalence Study of Infections in Urology 2013 revealed post-biopsy infection in $5.2 \%$ of patients; of them, $3 \%$ required hospitalisation. ${ }^{3}$ A recently published populationbased study showed an increasing trend in infective complications, comprising a four-fold increase in overall hospitalisations over 10 years. ${ }^{13}$ In the present study, we could not perform any temporal analyses of complications because the length of the study was insufficient; to the best of our knowledge, there have been no such temporal analyses in Hong Kong. The infection rate in our cohort was comparatively lower than that of most international studies, ${ }^{4}$ and similar to that in prior studies elsewhere in Asia ${ }^{14,15}$ ( $0 \%$ and $0.5 \%$ of PBI), as well as in Hong Kong ${ }^{5,6}(0.5 \%$ and $3.9 \%)$. Reasons for the apparent lower infection rate in people of Asian ethnicity compared with those of other ethnicities are unclear. Tsu et $\mathrm{al}^{6}$ reported that patients who underwent TRUS biopsy exhibited a high prevalence $(53.6 \%)$ of antibiotic-resistant flora in the rectum, although the PBI rate remained low among these patients (2.4\%). Numerous risk factors have been associated with the development of PBI. ${ }^{4}$ However, in the present study, we did not identify any factors that could predict the risk of PBI.

A positive urine culture was not a mandatory requirement to define $\mathrm{PBI}$ in this study, as a significant proportion of men who had urinary tract infection symptoms without systemic inflammatory response syndrome were treated and discharged directly from the emergency department, and most did not provide urine cultures. Thus, the emergency case notes were reviewed to determine whether PBI had occurred. In contrast, for men who had been hospitalised with sepsis, urine and blood cultures were available for analysis.

There were no reports of mortality in our cohort. In general, death directly related to biopsy is exceedingly rare and most patients die because of other factors. The reported mortality rates after TRUS biopsy are $0.09 \%$ to $1.3 \%$, depending on the length of the post-biopsy follow-up period. ${ }^{4}$ Data from a prostate cancer screening trial showed a mortality rate of $0.095 \%$ in biopsy patients, which was 
comparable to that of the control group. Notably, the mortality rate in biopsy patients was lower than that in patients who had no biopsies; none of the deaths in the study were related to the biopsy procedure. ${ }^{16}$

\section{Transperineal or transrectal approaches}

There has been a recent surge of interest, both in Hong Kong and internationally, in performing transperineal prostate biopsies. Transperineal biopsies are advantageous in that they have an extremely low risk of sepsis and enable improved sampling of tumours in the anterior prostate. ${ }^{17}$ In transperineal biopsy, the needle is passed through clean and prepared skin, rather than faeces or bowel; this method is presumed to eliminate post-biopsy infection. In 2013, a large systematic review of transperineal biopsy showed no instances of sepsis, with only a few reported cases of PBI (0\%-1.6\%). Transrectal biopsy exhibits difficulty in sampling the anterior prostate. Indeed, transperineal biopsy reportedly exhibits a superior cancer detection rate, especially in terms of tumours in the anterior prostate. ${ }^{18,19}$

Despite these advantages in the rate of postbiopsy sepsis and sampling of anterior tumours, the transperineal approach has limitations. These include longer operating time, greater procedurerelated pain, and increased post-biopsy retention, particularly in relation to the use of template mapping protocols. ${ }^{20,21}$ A systematic review and meta-analysis conducted in 2012, which compared the outcomes of transperineal and transrectal biopsies, did not show any differences in rates of complications between the two approaches. ${ }^{22}$ In our opinion, additional studies are needed to compare the two approaches in terms of cancer detection rate, complications, cost-effectiveness, and patient-reported outcomes before wide adoption of the transperineal approach is recommended.

In early 2018, we began exploratory use of transperineal prostate biopsy; thus far, we have used it for assessment of 71 patients. None of the patients have shown signs of sepsis or urinary tract infections; two patients were readmitted after biopsy for urethral bleeding and three patients were readmitted for urinary retention. The number of biopsies performed thus far is insufficient for a meaningful comparison with existing data from transrectal biopsies.

\section{Limitations and future studies}

To the best of our knowledge, this is the first study in Hong Kong to provide data regarding non-infective complications of TRUS biopsy. It provides valuable information for patients and can be used by clinicians during treatment counselling. Special precautions and education are needed for patients with a large prostate, as they exhibit an increased risk of postbiopsy retention. Nonetheless, the value of this study was limited by its retrospective nature.

The complications recorded were based solely on emergency attendances and hospitalisations in all public hospitals; importantly, attendances to private sector hospitals might have been missed. However, because approximately $90 \%$ of in-patient care in Hong Kong is provided by public hospitals, we presume that our approach enabled us to retrieve data regarding the vast majority of post-biopsy complications that required hospitalisations. ${ }^{23}$ In addition, patients who had attended private hospitals for complications, then attended public out-patient clinics for follow-up, could be identified and recorded unless they also selected private clinic follow-up.

Milder complications which did not require emergency attendances or hospitalisations, as well as sexual dysfunction and post-biopsy pain, could not be assessed in this study. Because of its retrospective design, we also could not report on prior antibiotics exposure and travel history among the patients, which limits analyses of risk factors. The number of cores taken could have affected the rate of complications. ${ }^{4}$ Approximately $20 \%$ of men in the cohort had sextant biopsies. The use of this lower number of cores might have led to underestimation of the rate of complications, compared with current standards for biopsy, in which 10 to 12 cores are taken.

Finally, a locoregional prospective multicentre study with other Asian nations would provide valuable insights into complications after prostate biopsies in the Asian population; it would also aid in assessments of differences in complications compared with Western nations.

\section{Conclusions}

Complications requiring emergency attendances or hospitalisations after transrectal prostate biopsy were uncommon; the most common complications requiring emergency attendances and hospitalisations were gross haematuria and AUR, respectively. Prostate volume $>48 \mathrm{cc}$ was a risk factor for post-biopsy urinary retention, but no specific risk factors were identified for post-biopsy infections. Patients with large prostate should be counselled for the increased risk of urinary retention after TRUS biopsy.

\section{Author contributions}

All authors had full access to the data, contributed to the study, approved the final version for publication, and take responsibility for its accuracy and integrity.

Concept or design: KC Cheng, KM Lam.

Acquisition of data: KC Cheng, WC Lam, KM Lam.

Analysis or interpretation of data: KC Cheng. 
Drafting of the article: KC Cheng.

Critical revision for important intellectual content: HC Chan, CC Ngo, MH Cheung, HS So.

\section{Declaration}

This research has been presented in part at the 15th Urological Association of Asia Congress 2017, 4-6 August 2017, Hong Kong.

\section{Conflicts of interest}

All authors have disclosed no conflicts of interest.

\section{Acknowledgement}

We acknowledge and express our gratitude to Dr YS Chan and Dr Alvin Chan for the data entry.

\section{Funding/support}

This research received no specific grant from any funding agency in the public, commercial, or not-for-profit sectors.

\section{Ethics approval}

This study was approved by the Kowloon Central/Kowloon East Research Ethics Committee (Ref KC/KE-19-0182/ER-1).

\section{References}

1. Hodge KK, McNeal JE, Stamey TA. Ultrasound guided transrectal core biopsies of the palpably abnormal prostate. J Urol 1989;142:66-70.

2. Prostate Cancer: Diagnosis and Treatment. Cardiff, UK: National Collaborating Centre for Cancer; 2014.

3. Wagenlehner FM, van Oostrum E, Tenke P, et al. Infective complications after prostate biopsy: outcome of the Global Prevalence Study of Infections in Urology (GPIU) 2010 and 2011, a prospective multinational multicentre prostate biopsy study. Eur Urol 2013;63:521-7.

4. Loeb S, Vellekoop A, Ahmed HU, et al. Systematic review of complications of prostate biopsy. Eur Urol 2013;64:87692.

5. Chan ES, Lo KL, Ng CF, Hou SM, Yip SK. Randomized controlled trial of antibiotic prophylaxis regimens for transrectal ultrasound-guided prostate biopsy. Chin Med J (Engl) 2012;125:2432-5.

6. Tsu JH, Ma WK, Chan WK, et al. Prevalence and predictive factors of harboring fluoroquinolone-resistant and extended-spectrum beta-lactamase-producing rectal flora in Hong Kong Chinese men undergoing transrectal ultrasound-guided prostate biopsy. Urology 2015;85:15-21.

7. von Elm E, Altman DG, Egger M, Pocock SJ, Gøtzsche PC, Vandenbroucke JP; STROBE Initiative. The Strengthening the Reporting of Observational Studies in Epidemiology (STROBE) statement: guidelines for reporting observational studies. J Clin Epidemiol 2008;61:344-9.

8. Singer M, Deutschman CS, Seymour CW, et al. The Third International Consensus Definitions for Sepsis and Septic Shock (Sepsis-3). JAMA 2016;315:801-10.

9. Rosario DJ, Lane JA, Metcalfe C, et al. Short term outcomes of prostate biopsy in men tested for cancer by prostate specific antigen: prospective evaluation within ProtecT study. BMJ 2012;344:d7894.

10. Raaijmakers R, Kirkels WJ, Roobol MJ, Wildhagen MF, Schrder FH. Complication rates and risk factors of 5802 transrectal ultrasound-guided sextant biopsies of the prostate within a population-based screening program. Urology 2002;60:826-30.

11. Zaytoun OM, Anil T, Moussa AS, Jianbo L, Fareed K, Jones JS. Morbidity of prostate biopsy after simplified versus complex preparation protocols: assessment of risk factors. Urology 2011;77:910-4.

12. Ghani KR, Dundas D, Patel U. Bleeding after transrectal ultrasonography-guided prostate biopsy: a study of 7-day morbidity after a six-, eight- and 12-core biopsy protocol. BJU Int 2004;94:1014-20.

13. Nam RK, Saskin R, Lee Y, et al. Increasing hospital admission rates for urological complications after transrectal ultrasound guided prostate biopsy. J Urol 2010;183:963-8.

14. Raheem OA, Casey RG, Galvin DJ, et al. Discontinuation of anticoagulant or antiplatelet therapy for transrectal ultrasound-guided prostate biopsies: A single-center experience. Korean J Urol 2012;53:234-9.

15. Shigemura K, Matsumoto M, Tanaka K, Yamashita M, Arakawa S, Fujisawa M. Efficacy of combination use of betalactamase inhibitor with penicillin and fluoroquinolones for antibiotic prophylaxis in transrectal prostate biopsy. Korean J Urol 2011;52:289-92.

16. Pinsky PF, Parnes HL, Andriole G. Mortality and complications after prostate biopsy in the Prostate, Lung, Colorectal and Ovarian Cancer Screening (PLCO) trial. BJU Int 2014;113:254-9.

17. Pepe P, Garufi A, Priolo G, Pennisi M. Transperineal versus transrectal MRI/TRUS fusion targeted biopsy: detection rate of clinically significant prostate cancer. Clin Genitourin Cancer 2017;15:e33-6.

18. Ong WL, Weerakoon M, Huang S, et al. Transperineal biopsy prostate cancer detection in first biopsy and repeat biopsy after negative transrectal ultrasound-guided biopsy: the Victorian Transperineal Biopsy Collaboration experience. BJU Int 2015;116:568-76.

19. Huang S, Reeves F, Preece J, Satasivam P, Royce P, Grummet JP. Significant impact of transperineal template biopsy of the prostate at a single tertiary institution. Urol Ann 2015;7:428-32.

20. Guo LH, Wu R, Xu HX, et al. Comparison between ultrasound guided transperineal and transrectal prostate biopsy: a prospective, randomized, and controlled trial. Sci Rep 2015;5:16089.

21. Skouteris VM, Crawford ED, Mouraviev V, et al. Transrectal ultrasound-guided versus transperineal mapping prostate biopsy: complication comparison. Rev Urol 2018;20:19-25.

22. Shen PF, Zhu YC, Wei WR, et al. The results of transperineal versus transrectal prostate biopsy: a systematic review and meta-analysis. Asian J Androl 2012;14:310-5.

23. Kong X, Yang Y, Gao J, et al. Overview of the health care system in Hong Kong and its referential significance to mainland China. J Chin Med Assoc 2015;78:569-73. 